\title{
How lost "passenger" ants find their way home
}

\author{
Mandyam V. Srinivasan ${ }^{1}$
}

Published online: 1 May 2017

(C) Psychonomic Society, Inc. 2017

Summary Animal navigation has fascinated biologists and engineers for centuries, and some of the most illuminating discoveries have come from the study of creatures with a brain no larger than a sesame seed. In an elegant recent study, Pfeiffer and Wittlinger (Science, 353, 1155-1157, 2016) have shown the means by which desert ants, carried from one nest to another by a relative, find their own way back home if they are accidentally dropped en route.

Keywords Navigation · Orientation · Ant · Dead Reckoning

The species under study was the desert ant Cataglyphis, whose navigational abilities were first documented early in the 19th century, and subsequently investigated quantitatively over several decades in a series of trail-blazing studies initiated by the laboratory of Rüdiger Wehner. Individual Cataglyphis foragers set out from their nest and carry out a meandering search, looking for food in the form of an insect or spider that has expired in the extreme heat of the desert sun. When a forager discovers a food item, it picks up the bounty and runs directly back to the nest in a straight line (Wehner, Cheng, \& Cruse, 2014). A series of ingenious experiments have demonstrated that these desert ants are capable of navigating in featureless terrain, devoid of landmarks, by using a process of "path integration." (Most desert ants do not rely on pheromone trails, which evaporate in the heat.) Path integration involves keeping track of the distance and direction

Mandyam V. Srinivasan

m.srinivasan@uq.edu.au

1 Queensland Brain Institute and School of Information Technology and Electrical Engineering, University of Queensland, St.

Lucia, QLD 4072, Australia of travel along each segment of the tortuous outbound journey, adding up the vectors representing the movements along the individual segments, and using the resultant vector to keep a running record of the distance and direction of the ant's current position relative to the nest. To perform this navigational feat, the ant would need to rely on a compass to record the direction of travel, and an odometer to measure the distance traveled. Desert ants obtain compass information from the position of the sun and the pattern of polarization that it creates in the sky (Wehner et al., 2014). Foraging ants perform odometry by counting footsteps or, more precisely, by integrating the movements of the legs over time (Wehner et al., 2014).

Honeybees are the other insect in which navigation has been investigated extensively. Like desert ants, honeybees use a sky compass (Wehner et al., 2014). Initially, it was thought that bees measure distance traveled by recording the energy consumed during the flight. However, more recent studies indicate that odometry is performed visually by measuring the extent to which the image of the environment has moved in the eyes during the journey (Srinivasan, 2011).

This background of previous research on desert ants and honeybees sets the context for the question now posed by Pfeffer and Wittlinger (2016), who investigated the behavior of ants that are carried from one nest to another by a nest mate. How does an ant, being transported from one nest to another by an experienced carrier, cope when it is accidentally dropped and lost en route? The authors set up a channel connecting two nests, $A$ and $B$, ca. $15 \mathrm{~m}$ apart, between which ants were often observed to carry nest mates from one nest to the other. The channels had walls but were open at the top, allowing the ants a view of the sky. After the ants were accustomed to using the channel to walk between the nests, individual ants that were being carried from $A$ to $B$ were tested as follows. The carrier ant and the carried ant were separated from each other after they had traveled a distance of 10 $\mathrm{m}$ from $A$. The carried ant was then released in a fresh test tunnel, 
oriented in the same direction as the original tunnel, and its behavior was observed. The carried ant ran in the direction toward nest $A$, the nest from which the pair had emerged, and searched for the nest by zig-zagging back and forth at a distance of approximately $10 \mathrm{~m}$ from the release point, namely, at the expected position of the nest. The carrier ant, on the other hand, proceeded toward nest $B$. This remarkable finding indicates that a carried ant is capable of registering how far it has been transported and returning to the home nest, even if it has not walked the journey on its own. How is this achieved? When the ventral regions of the eyes of the carried ant were occluded with paint before the carrier-carried pair commenced its outbound journey, and the carried ant was separated from the carrier when they had reached the $10 \mathrm{~m}$ point, the carried ant was no longer able to find its way home - regardless of whether the paint was left on or removed. This ingenious experiment demonstrated that the carried ant estimates the distance over which it has been carried by integrating the motion of the image of the ground that it has experienced during the outbound journey.

Pfeffer and Wittlinger (2016) posed a further question: If the carried ant experiences image motion on the outbound journey but is deprived of this visual input when it is released (by painting the ventral regions of the eyes as described above), is it able to transfer the information acquired by its visual odometer to its "pedometer" and find its way back home by counting its footsteps? Apparently not—carried ants treated in this way are disoriented when they are released. Thus, while foraging individuals largely use pedometry to measure their travel distance, carried ants rely on visual odometry.

The observation that a carried Cataglyphis ant can find its way back home was published more than 40 years ago in two landmark papers by Peter Duelli (Duelli, 1976, and another paper cited therein), which showed that (a) carried ants head homeward after they are separated from their carriers, and (b) the carried ant runs back a distance that corresponds approximately to the distance from the nest at which it was separated from the carrier on the outbound journey. The directional orientation of the carried ants was also confirmed by Fourcassie, Abdallah Dahbi, and Xim (2000).

The present study takes these findings one step further by demonstrating that the carried ant uses vision to measure its travel distance on its outbound as well as its return journey. This is probably the first clear demonstration of the use of a purely vision-based odometer by a walking insect. With flying insects, visual odometry is complicated by the fact that the optic flow generated by the ground depends upon the height of flight - the greater the height, the lower the magnitude of the optic flow (Srinivasan, 2011). This problem does not arise with a walking insect, because the eyes are at an approximately constant distance from the ground. Thus, it would appear that walking insects are very well placed for performing visual odometry; which begs the question why Cataglyphis has apparently evolved two systems for measuring travel distancepedometry for foragers and visual odometry for carried ants.

The new findings raise at least two further intriguing questions:

(i) In the current study, training and testing were carried out in straight tunnels. This meant that, in the outbound journey, the carried ant did not need to perform the kind of complex path integration that a foraging ant typically performs during its tortuous outbound journey in search of food. Carried ants probably experience nearly linear trajectories even in natural situations because the carrier is already familiar with the location of the destination nest and runs directly toward it. It would be interesting to examine whether carried ants are capable of performing complex path integration by training a carrier/carried pair to navigate from one nest to another through an $L$-shaped tunnel and observing the behavior of the carried ant when it is separated from the carrier at the end of the tunnel. If the carried ant heads back home along a direction parallel to the hypotenuse of the $L$, and searches for the nest at a distance corresponding to the length of the hypotenuse, this would imply that the carried ant can indeed use its visual odometer and sky compass to perform complex, vector-based path integration.

(ii) What is the neural basis of complex path integration in these tiny brains? How are the instantaneous directions and speeds of travel encoded at the neural level and combined and integrated vectorially to achieve this impressive feat? Progress toward answering this question is now beginning to be made in walking as well as flying insects.

\section{References}

Duelli, P. (1976). Distanzdressuren von getragenen Ameisen (Cataglyphis bicolor) [Distance training of carried ants (Cataglyphis bicolor)]. Revue Suisse de Zoologie, 83, 413-418.

Fourcassie, V., Abdallah Dahbi, A., \& Xim, C. (2000). Orientation and navigation during adult transport between nests in the ant Cataglypis iberica. Naturwissenschaften, 87, 355-359.

Pfeffer, S. E., \& Wittlinger, M. (2016). Optic flow odometry operates independently of stride integration in carried ants. Science, 353, $1155-1157$.

Srinivasan, M. V. (2011). Honeybees as a model for the study of visually guided flight, navigation, and biologically inspired robotics. Physiological Reviews, 91, 389-411.

Wehner, R., Cheng, K., \& Cruse, H. (2014). Visual navigation strategies in insects: Lessons from desert ants. In J. S. Werner \& L. M. Chalupa (Eds.), The new visual neurosciences (pp. 1153-1164). Cambridge, MA: MIT Press. 